\title{
Manufacturing and mechanical properties of composite orthotropic Kagome honeycomb using novel modular method
}

\author{
(C) The Author(s) 2020. This article is published with open access at link.springer.com and journal.hep.com.cn
}

\begin{abstract}
This work deals with manufacturing and analysis of orthotropic composite Kagome honeycomb panels. A novel modular mold is proposed to manufacture carbon fiber reinforced composite Kagome honeycombs. The designed mold can be assembled freely to manufacture Kagome honeycombs with different configuration combinations and can realize easy demolding. Furthermore, two typical fiber placement methods are considered during the fabrication process, from which the more effective fiber placement method is determined. Finally, representative volume element method is used to perform homogenization analysis of the Kagome honeycomb panels and to obtain equivalent in-plane and bending stiffness. Finite element analysis using these equivalent properties is conducted and validated against the experimental results of the manufactured composite Kagome honeycomb panels under different loading cases.
\end{abstract}

Keywords composite, Kagome honeycomb, manufacturing, placement of fibers, equivalent stiffness

\section{Introduction}

Composite honeycombs have excellent mechanical properties, such as low weight, high strength, and high loadcarrying capacity; they have been widely used in many industrial fields in recent years $[1,2]$. Currently, typical honeycomb structures include hexagonal honeycomb, triangular honeycomb, and Kagome honeycomb [3-7]. Compared with other honeycombs, a composite Kagome honeycomb consists of hexagonal and triangular configurations; it is hybrid honeycomb and has balanced in-plane

Received November 18, 2019; accepted April 20, 2020

Bin NIU, Shijie LI, Rui YANG ( $₫)$

Key Laboratory for Precision and Non-Traditional Machining Technology of Ministry of Education, School of Mechanical Engineering, Dalian University of Technology, Dalian 116024, China E-mail: yangrui@dlut.edu.cn and out-of-plane properties. Different from bendingdominated foam, stretching-dominated Kagome honeycombs have relatively high weight efficiency [8]. This finding is caused by the difference between composite Kagome honeycomb and other types of honeycomb in the fabricating method, mechanical properties, and application. Therefore, Kagome honeycomb has attracted remarkable attention of researchers as a novel ultralight multifunctional structural material.

Kagome honeycomb is one of the most attractive honeycomb configurations, and many methods are developed for its design. Deshpande et al. [8] found that Kagome honeycomb is a stretching-dominated honeycomb. They presented the topology design criterion of 2D honeycomb. Totaro and Gürdal [9] proposed a Kagome honeycomb optimization method for realizing minimum mass solution under buckling and strength constraints. Niu et al. [10] developed a two-scale topology optimization method to obtain optimum macrostructure and material microstructure for maximum fundamental frequency. The optimum microstructures are found to be Kagome configurations in many cases.

Manufacturing process of metal Kagome honeycomb, such as investment casting [11] and powder extrusion molding [12], is already mature. However, manufacturing of composite Kagome honeycomb is still a key factor in restricting its wide application. Vasiliev et al. [13] introduced the history, preparation, and application of composite honeycombs. Sorrentino et al. [14] designed rib dimensions of composite isogrid structure and fabricated the structure using an epoxy mold. Han and Tsai [15] used interlocking process with pultruded glass fiber ribs to manufacture honeycomb panels. They also used unidirectional fibers, which can be aligned along the rib directions, to fully utilize the mechanical properties of fibers. Fan et al. [16] prepared a composite Kagome grid sandwich panel by interlocking process. They conducted in-plane compression, out-of-plane compression, and three-point bending experiments. Their results showed that Kagome grid sandwich panels have higher specific strength and specific 
stiffness than other types of sandwich panels.

The mechanical properties of Kagome honeycombs, which are hybrid honeycombs, are more complex than other types of honeycombs. Wang and McDowell [17] derived parametric expressions of effective properties of isotropic Kagome honeycombs from the discrete mesoscopic truss model. Niu and Wang [18] investigated the inplane mechanical properties of periodic honeycomb structures with orthotropic Kagome cells using the truss model of Wang and McDowell [17]. Cheng et al. [19,20] introduced a novel numerical implementation of asymptotic homogenization method to predict effective properties of periodic materials. The method can be implemented using finite element analysis software as a black box. Peng and $\mathrm{Yu}$ [21] applied novel theory of structure genome to obtain effective properties of heterogeneous materials. They built a connection between the macroscopic structures and the heterogeneous material properties. Liu and $\mathrm{Su}$ [22] introduced an effective couple-stress continuum model using the representative volume element (RVE) method to describe the properties of cellular material. The discrete analysis of the composite Kagome honeycomb is more complicated and incurs a high computational cost because the composite is orthotropic or anisotropic. Therefore, using homogenization method is a good alternative to obtain equivalent performance of the honeycomb structure.

Inspired by these studies, the aim of the present study is to design, manufacture, and analyze Kagome honeycomb panels made of carbon fiber reinforced polymer (CFRP). The mold design, fiber placement method, forming technology, theoretical prediction of effective properties, and experiments are investigated. A new modular mold achieving integral forming of the honeycombs is designed in the present work for the Kagome honeycomb. The designed mold can be assembled freely to manufacture Kagome honeycombs with different configuration combinations. Meanwhile, the influences of typical fiber placement methods on the mechanical properties of the structure are compared. Furthermore, a homogenization analysis model is developed using RVE to calculate the equivalent stiffness of orthotropic composite Kagome honeycomb panel, and finite element analyses using these equivalent properties are conducted. Then, experiments of the composite Kagome panels under different loading cases are carried out to validate the predicted equivalent properties of orthotropic composite Kagome honeycomb panel. The present manufacturing approach based on the novel modular mold can be extended and applied to fabricate composite honeycomb panels with various cell configurations. Among many other applications of the manufactured composite Kagome honeycomb panels, aircraft sandwich constructions can use the honeycomb panel as the core to improve lightness and increase loadcarrying capacity.

The contents of the present article are organized as follows. In Section 2, we design a Kagome honeycomb modular mold and conduct a vacuum bag assisted curing process to prepare Kagome honeycombs with different combinations. Two different fiber placement methods are used in the preparation, and the effects of these methods on the mechanical properties of the Kagome honeycombs are compared experimentally. Material parameters of the composite used in fabricating Kagome honeycombs are measured experimentally. These parameters will be used for subsequent theoretical analysis. In Section 3, the equivalent stiffness of the Kagome honeycombs is calculated by RVE, and the calculated equivalent stiffness is verified by the discrete model analysis. In Section 4, the in-plane and out-of-plane mechanical properties of the prepared Kagome honeycombs are tested and compared with the predicted results. Section 5 presents the conclusions and discussion.

\section{Manufacturing orthotropic composite Kagome honeycomb}

\subsection{Mold design}

First, inspired by the results of the optimized designs [10], the shape and size of the Kagome honeycomb panels are determined. The periodic orthotropic honeycomb structure with its dimensions is shown in Fig. 1; the out-of-plane
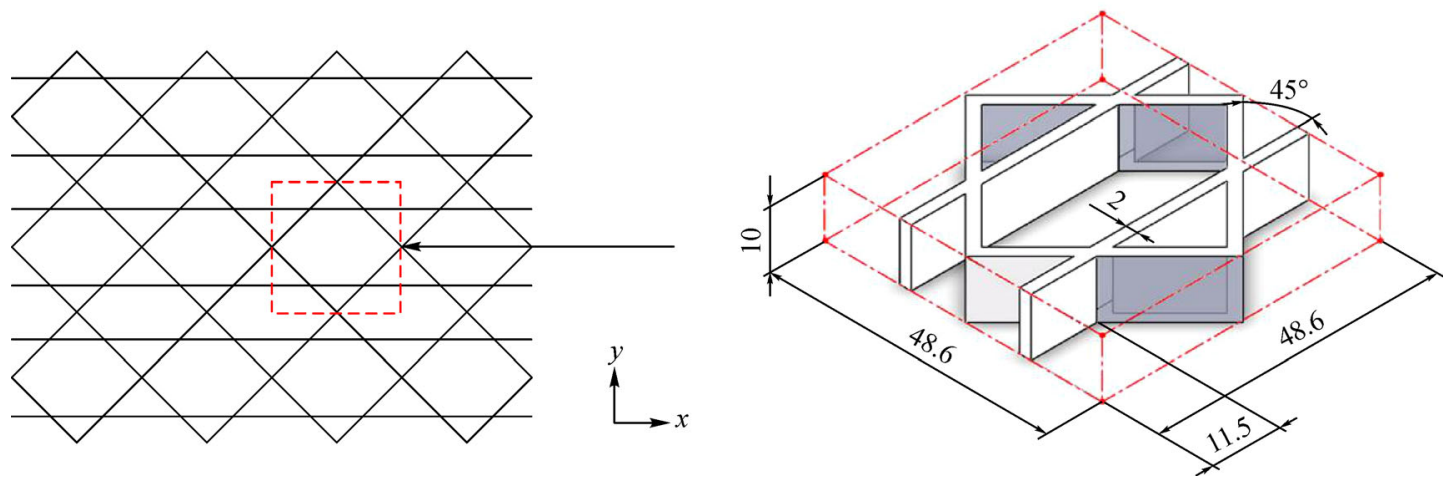

Fig. 1 Schematic of Kagome honeycombs (unit: $\mathrm{mm}$ ). 
height of the structure is $10 \mathrm{~mm}$.

Second, we design and manufacture a modular mold of Kagome honeycomb panels, as shown in Fig. 2. The designed mold is composed of a metal base plate, some internal blocks, and boundary blocks as shown in Fig. 2(a). The internal and boundary blocks can be assembled and fixed through the positioning slots, which are on the base plate. The internal blocks are assembled on the metal base plate in accordance with the number of rows and columns of the unit cells to be produced, and the boundary blocks are used to form the edge of the internal blocks. We can use the same mold to manufacture Kagome honeycomb panels with different configuration combinations through different combinations of internal and boundary blocks. Gaps exist between the adjacent internal blocks and the boundary blocks, and these gaps with Kagome geometry form fiber placement gaps. This modular mold can be assembled freely to manufacture Kagome honeycomb panels with different combinations. In addition, easy

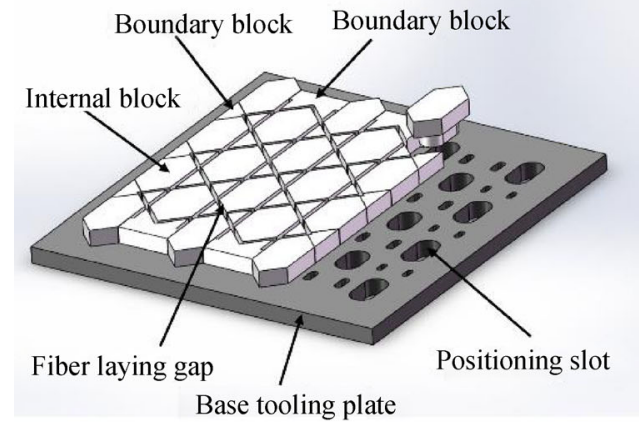

(a) demolding can be realized by removing the boundary and internal blocks. More importantly, the designed mold can achieve integral forming of the Kagome honeycomb panels. The manufactured modular mold is shown in Fig. 2(b).

\subsection{Manufacturing process}

The manufacturing process of composite Kagome honeycomb is shown in Fig. 3. The specific process is as follows: First, the release agent is evenly applied on the internal blocks, the boundary blocks, and the base plate to demold easily. Then, the designed mold is assembled according to the Kagome honeycomb combination to be manufactured. Second, epoxy resin (LY5288) and curing agent (HY5289) in a mass ratio of 5:1 are mixed uniformly. Then, the mixture is evenly smeared on the carbon fiber bundle (T300-3k) to obtain a carbon fiber prepreg bundle, which will be placed in the fiber laying gap in a certain placement

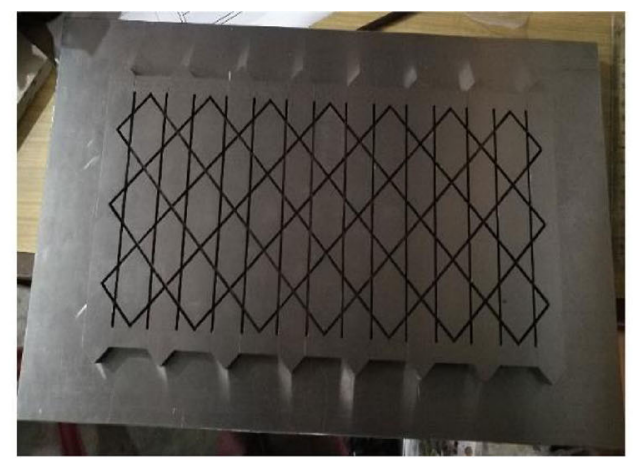

(b)

Fig. 2 Designed modular mold. (a) Mold diagram; (b) actual photo of the mold.

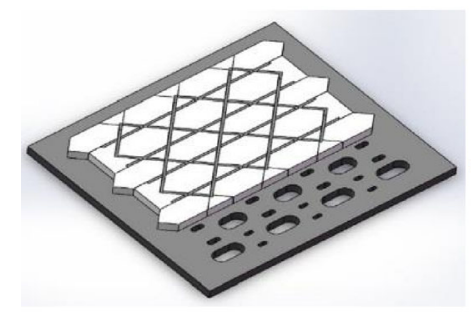

(a)

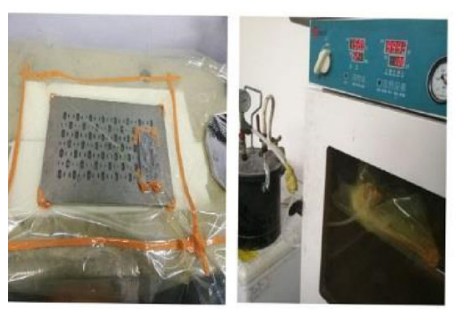

(c)

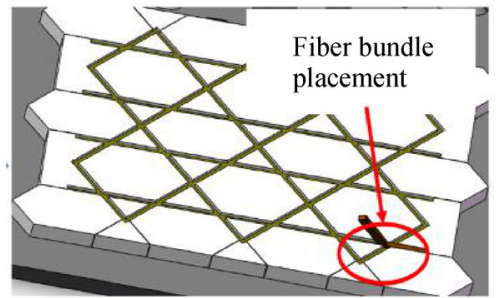

(b)

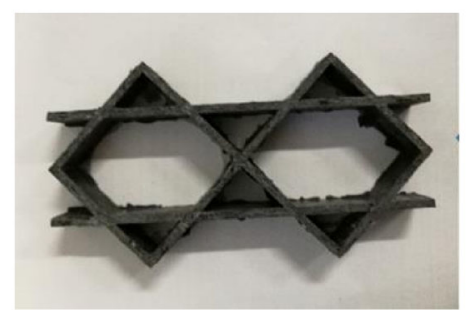

(d)

Fig. 3 Manufacturing process of Kagome honeycombs. (a) Assemble mold, (b) fiber placement, (c) vacuum bag assistance and heat, and (d) Kagome honeycomb $(2 \times 1)$. 
method (see further detailed discussion). Third, the double vacuum bag is used to carry out compaction operation with a pressure of $0.1 \mathrm{MPa}$. The mold is heated to $150^{\circ} \mathrm{C}$ for $4 \mathrm{~h}$ to cure. Fourth, the internal and boundary blocks are removed to achieve mold release, and the cured resin remaining on the edge of the honeycomb is trimmed.

The placement of the fibers has a crucial influence on the overall mechanical properties of the structure. Therefore, to determine the more effective fiber placement, we have compared two typical methods of placements.

Case A: Cascading laying, as plotted in Fig. 4. To introduce the fiber placement in details, the two straight sides are labeled as $\mathrm{S} 1$ and $\mathrm{S} 2$, and the oblique side is labeled as O1, as shown in Fig. 4(a). Two fiber bundles are piled into S1 and S2, shown as the two red fibers in the first layer and marked as S1-1 and S2-1. Then, another relatively long fiber bundle is placed in $\mathrm{O} 1$, which is marked as the blue fiber in the bottom layer and named as O1-1. When the placement of the first layer is completed, the fiber placement for the second layer is performed similarly. In the placement of the second layer, the straight fiber bundle is marked in yellow and the oblique fiber in green. These fiber bundles are similarly named as S1-2,
S2-2, and O1-2. The layers are continuously placed using this fiber laying method until the height of the fibers in the gap is higher than the mold. Figure 4(b) shows how the fiber bundle accumulates and bends at the intersection of the Kagome configuration.

Case B: Segmented laying, as plotted in Fig. 5. Initially, the oblique side $\mathrm{O} 1$ is divided into several parts to ensure no intersection between any two parts. Only the fibers in the straight sides should remain continuous. As shown in Fig. 5, O1 has nine parts, marked from $\mathrm{A}$ to I. The placement for each fiber layer is the same. Two long fibers are placed into S1 and S2. Then, the long fibers are cut into corresponding lengths of other parts, which are placed into corresponding parts until all parts are covered by one layer. Similarly, the second, third, and fourth layers are placed into the fiber laying gap, and the process is completed when the gap is crammed. Evidently, no fiber bending and accumulation occur at each intersection. Instead, the ribs at the intersections are connected by resin.

To guide our subsequent Kagome honeycomb panel preparation, we perform mechanical experiments of the honeycombs manufactured using the two placement methods to determine which is more effective. The

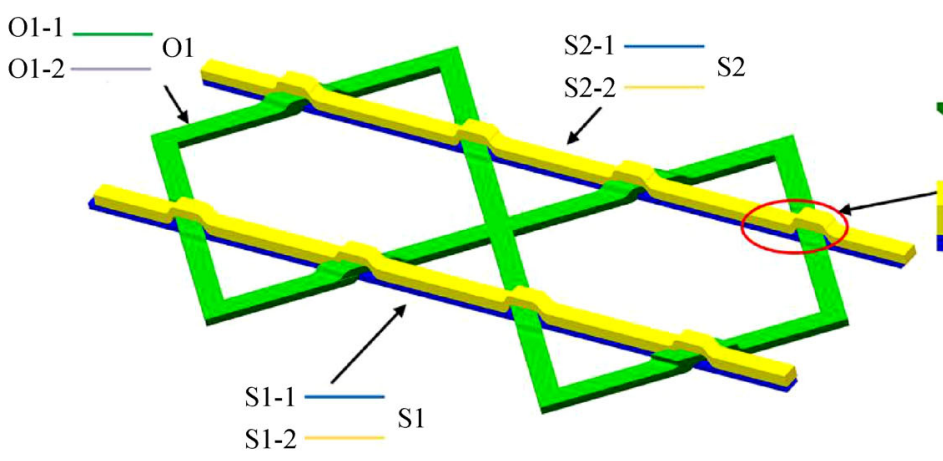

(a)

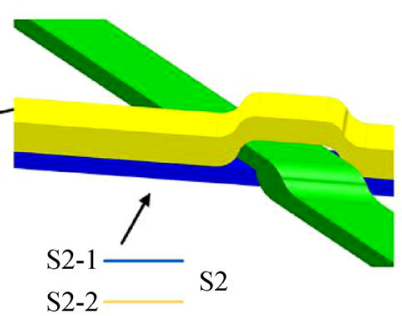

(b)

Fig. 4 Case A laying method. (a) Overall placement; (b) enlarged view of the intersection.

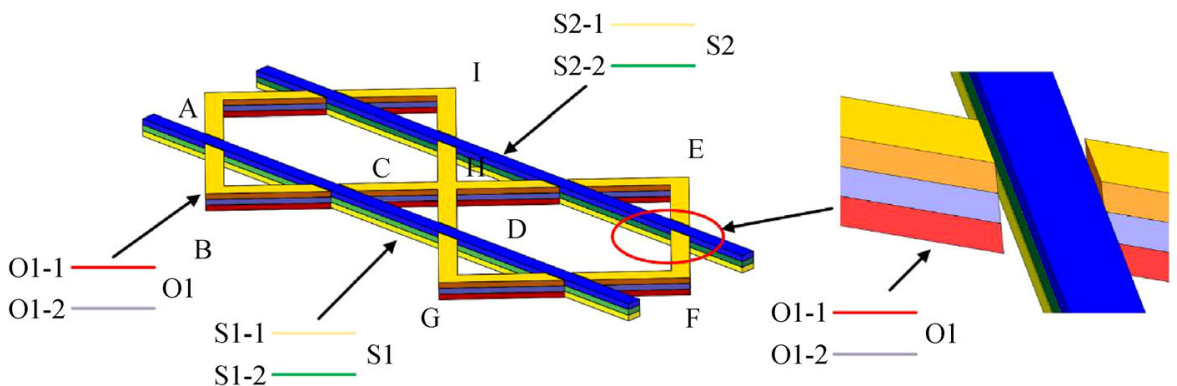

(a)

(b)

Fig. 5 Case B laying method. (a) Overall placement; (b) enlarged view of the intersection. 


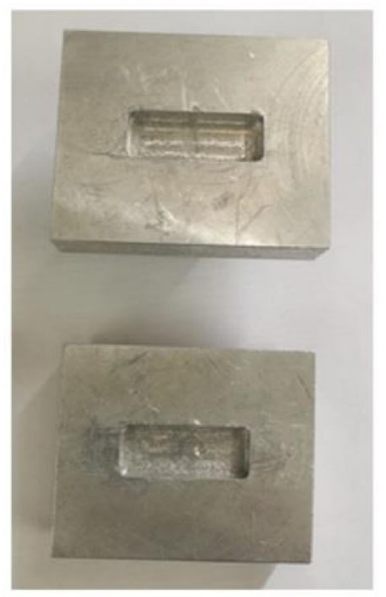

(a)

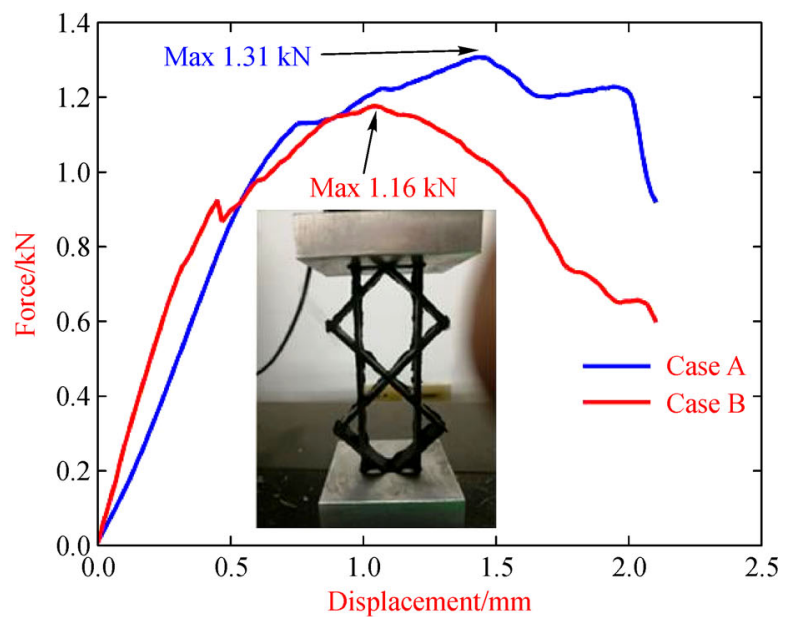

(b)

Fig. 6 Compression response comparison of Kagome honeycombs $(2 \times 1$ unit cells $)$ manufactured using the two placement methods: Cases A and B. (a) Testing fixture; (b) compression response of honeycomb.

Kagome honeycomb panels with $2 \times 1$ unit cells are used for in-plane compression tests. To prevent sliding of the top and bottom of the honeycomb during the experiment, a fixture, as shown in Fig. 6(a), is designed and used. Compression tests are performed on a testing machine with a displacement rate of $0.50 \mathrm{~mm} / \mathrm{min}$ to explore the mechanical properties of Kagome honeycombs. The compressive sample has a length of $93.8 \mathrm{~mm}$, width of $48.6 \mathrm{~mm}$, and height of $10 \mathrm{~mm}$. At least three samples are tested for the same placement. The typical force and displacement curves of the honeycombs obtained from the compression test are shown in Fig. 6(b), where the compression is carried out along the direction of the longer side (longitudinal direction). In the initial stage of loading, the force and displacement curves of the structure have a good linear relationship. In addition, the loadcarrying capacity of the honeycomb of Case A placement method starts to decrease after reaching $1.31 \mathrm{kN}$, and the honeycomb of Case B placement method reaches $1.16 \mathrm{kN}$. Experimental comparison shows that the honeycombs with two different fiber placement methods have slight difference in the in-plane stiffness in the longitudinal direction. However, the initial damage of the honeycombs of Case A placement method occurs later than that of Case B.

Subsequently, Kagome honeycomb panels of $3 \times 2$ unit cells are used for out-of-plane bending tests at a loading rate of $1 \mathrm{~mm} / \mathrm{min}$. Similarly, at least three samples are used for each placement method. The force and displacement curves of the honeycombs obtained from the bending test are shown in Fig. 7. Similarly, in the initial stage of loading, the force and displacement curves of the structure have a good linear relationship, and the load-carrying capacity of the honeycomb using Case A placement method starts to decrease after reaching $2.1 \mathrm{kN}$. However, the honeycomb using Case B placement method only

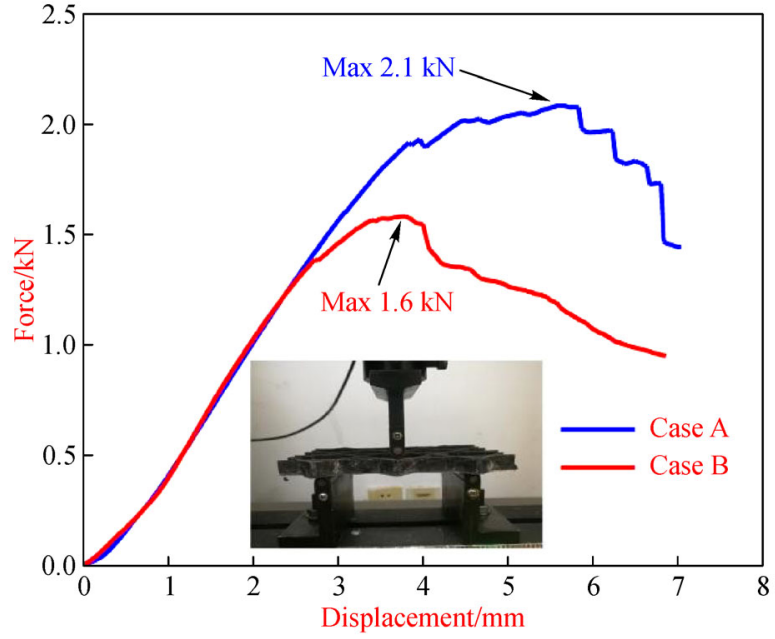

Fig. 7 Bending response comparison of Kagome honeycombs $(3 \times 2$ unit cells) manufactured using the two placement methods: Cases A and B.

reaches $1.6 \mathrm{kN}$. Evidently, honeycombs using Case A placement method have significantly better out-of-plane performance, which is different from the contrast of the compression response in Fig. 6(b). When the structure is subjected to bending, a part of the fibers is in compressed state and a part of the fibers is in stretched state. As a result, the condition of continuous or discontinuous fibers will largely affect the bending performance of the structure. The fibers in the two straight sides S1 and S2 shown in Figs. 4 and 5, respectively, are continuous in two cases, thereby explaining the slight difference in the compression along the longitudinal direction in Fig. 6(b). The difference in the transverse direction from the two placement methods become more evident due to the discontinuity at the intersection. 
The comparison of these experiments shows that Case A placement method can better use the mechanical properties of fibers. Case A placement method can ensure that the honeycomb has a continuous fiber connection at the intersection, and the honeycombs using Case A method have better mechanical properties. Therefore, in the subsequent fiber placement, we adopt Case A placement method.

\subsection{Mechanical properties of the material}

In this subsection, the mechanical properties of the cell wall are tested for subsequent theoretical analysis. The cell wall samples, with dimension of $45 \mathrm{~mm} \times 10 \mathrm{~mm} \times 2 \mathrm{~mm}$, are manufactured using the modular mold. The elastic modulus of the cell wall in the longitudinal direction and transverse direction is tested separately. Prior to the experiment, the ends of the samples must be polished until smooth and should be placed in the special fixture. The force and displacement curves are shown in Fig. 8.

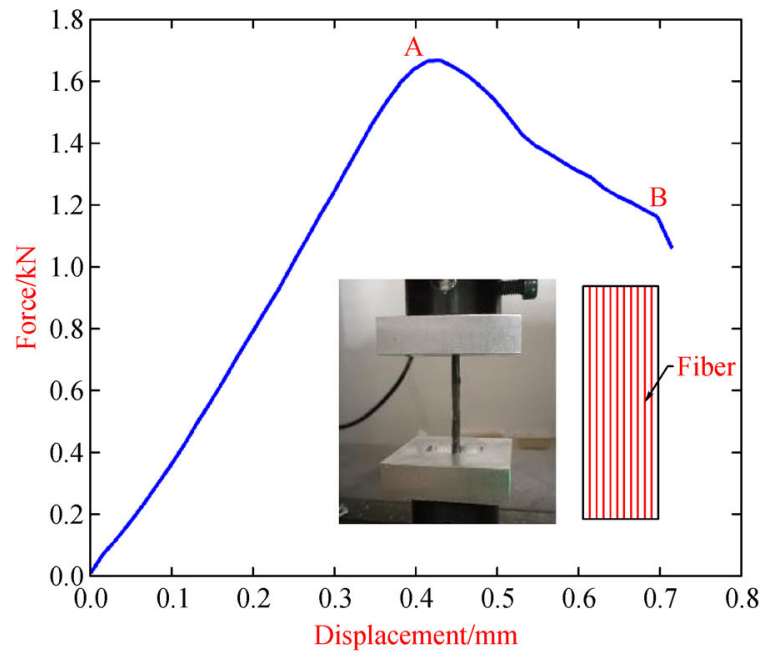

(a)
In addition, the Poisson's ratio and shear modulus of the material of the cell wall are measured by corresponding standard samples, following the test standards in GB/ T1447-2005 and GB/T3355-2005. The prepared standard samples and test processes are shown in Fig. 9. Five standard samples are prepared for each set of experiments. The mechanical properties of the materials obtained from the testing in each group are averaged, as shown below:

Longitudinal Young's modulus: $E_{11}=10.9 \mathrm{GPa}$;

Transverse Young's modulus: $E_{22}=E_{33}=3.47 \mathrm{GPa}$;

Poisson's ratio: $v_{12}=v_{13}=v_{23}=0.33$;

Shear modulus: $G_{12}=G_{13}=G_{23}=0.92 \mathrm{GPa}$.

\section{Homogenization analysis}

\subsection{ABD matrix and representative volume element}

A Kagome honeycomb panel is formed by periodically repeating the unit cell in the plane. The honeycomb has

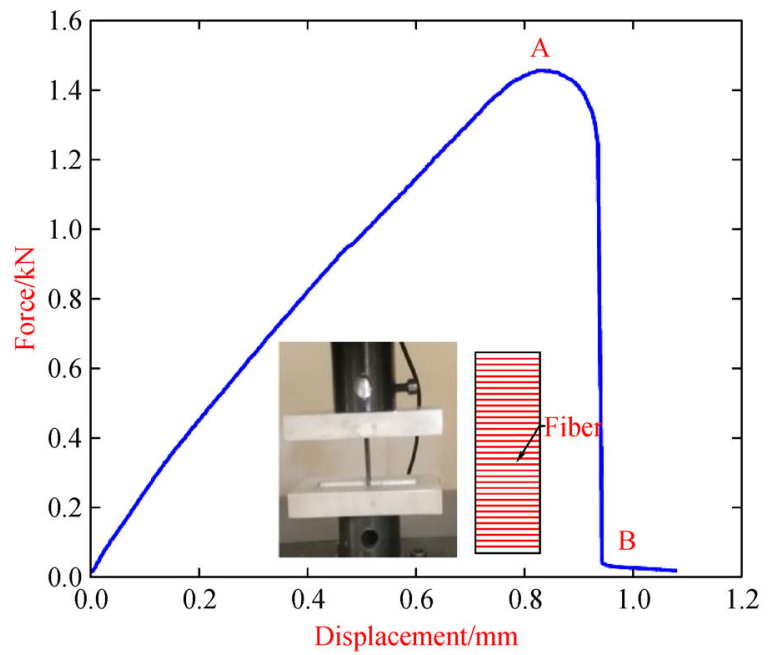

(b)

Fig. 8 Axial compression experiment of cell wall. (a) Direction 1 compression; (b) Direction 2 compression.

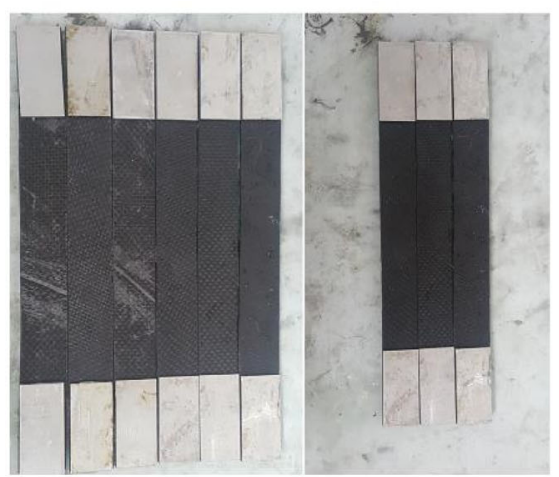

(a)

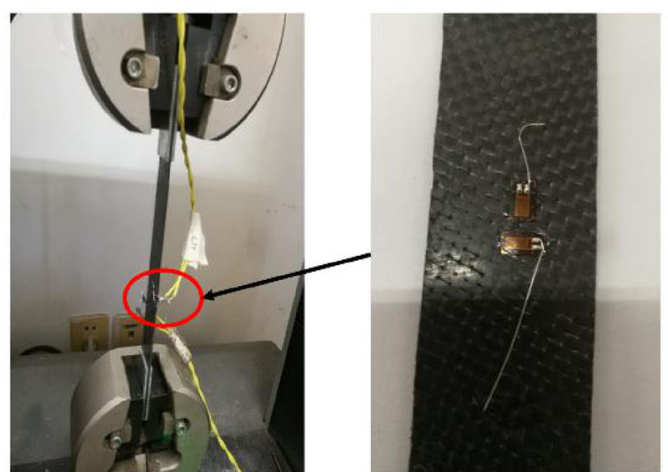

(b)

Fig. 9 Standard samples and test process of the shear modulus and Poisson's ratio. (a) Standard specimens; (b) test process. 
in-plane periodicity but no periodicity in the thickness direction. The deformation of the honeycomb panel may have evident characteristics of bending deformation because the size of the Kagome honeycomb panel in the thickness direction is much smaller than the dimensions in the other two directions. Therefore, the out-of-plane effective properties should be determined in addition to the in-plane effective properties. RVE method [22] is used to predict the effective properties of the honeycomb structures. The analysis of the unit cell of the honeycomb is conducted by taking into account the discrete characteristics of the honeycomb. Kagome honeycomb panel is homogenized into an equivalent classical laminate plate with effective stiffness coefficients $\boldsymbol{A}, \boldsymbol{B}$, and $\boldsymbol{D}$ using the RVE method, as shown in Eq. (1):

$$
\begin{aligned}
{\left[\begin{array}{l}
N_{x} \\
N_{y} \\
N_{x y} \\
M_{x} \\
M_{y} \\
M_{x y}
\end{array}\right]=\left[\begin{array}{llllll}
A_{11} & A_{12} & A_{16} & B_{11} & B_{12} & B_{16} \\
A_{12} & A_{22} & A_{26} & B_{12} & B_{22} & B_{26} \\
A_{16} & A_{26} & A_{66} & B_{16} & B_{26} & B_{66} \\
B_{11} & B_{12} & B_{16} & D_{11} & D_{12} & D_{16} \\
B_{12} & B_{22} & B_{26} & D_{12} & D_{22} & D_{26} \\
B_{16} & B_{26} & B_{66} & D_{16} & D_{26} & D_{66}
\end{array}\right]\left[\begin{array}{c}
\varepsilon_{x}^{0} \\
\varepsilon_{y}^{0} \\
\gamma_{x y}^{0} \\
\kappa_{x} \\
\kappa_{y} \\
\kappa_{x y}
\end{array}\right] } \\
= \\
{\left[\begin{array}{ll}
\boldsymbol{A} & \boldsymbol{B} \\
\boldsymbol{B}^{\mathrm{T}} & \boldsymbol{D}
\end{array}\right]\left[\begin{array}{c}
\varepsilon_{x}^{0} \\
\varepsilon_{y}^{0} \\
\gamma_{x y}^{0} \\
\kappa_{x} \\
\kappa_{y} \\
\kappa_{x y}
\end{array}\right] . }
\end{aligned}
$$

$\boldsymbol{N}=\left\{N_{x}, N_{y}, N_{x y}\right\}$ and $\boldsymbol{M}=\left\{M_{x}, M_{y}, M_{x y}\right\}$ represent internal forces and internal moments per unit length in the middle plane of the plate, respectively. The superscript $T$ denotes the transpose of a matrix or vector. The mid-plane strains and curvatures of the equivalent plate are given by $\varepsilon_{x}^{0}, \varepsilon_{y}^{0}, \gamma_{x y}^{0}, \kappa_{x}, \kappa_{y}$, and $\kappa_{x y} . \varepsilon_{x}^{0}=\partial u / \partial x, \varepsilon_{y}^{0}=\partial v / \partial y, \gamma_{x y}^{0}=$ $\partial u / \partial y+\partial v / \partial x, \quad \kappa_{x}=-\partial^{2} w / \partial x^{2}, \kappa_{y}=-\partial^{2} w / \partial y^{2}$, and $\kappa_{x y}=-2 \partial^{2} w / \partial x \partial y$, where the displacements in the three directions are denoted by $u, v$, and $w$. The middle $6 \times 6$ matrix is called the structured ABD matrix, which is symmetric. $\boldsymbol{A}, \boldsymbol{B}$, and $\boldsymbol{D}$ represent the in-plane stiffness, coupling stiffness, and bending stiffness matrix of the plate, respectively. The coupling stiffness matrix $\boldsymbol{B}$ is $\mathbf{0}$ because the Kagome honeycomb is orthotropic and symmetric. Furthermore, the in-plane stiffness matrix $\boldsymbol{A}$ and the bending stiffness matrix $\boldsymbol{D}$ have the following characteristics [23]:

$$
\begin{aligned}
& {\left[\begin{array}{l}
N_{x} \\
N_{y} \\
N_{x y}
\end{array}\right]=\left[\begin{array}{ccc}
A_{11} & A_{12} & 0 \\
A_{12} & A_{22} & 0 \\
0 & 0 & A_{66}
\end{array}\right]\left[\begin{array}{c}
\varepsilon_{x}^{0} \\
\varepsilon_{y}^{0} \\
\gamma_{x y}^{0}
\end{array}\right]=\boldsymbol{A} \boldsymbol{\varepsilon}^{0},} \\
& {\left[\begin{array}{l}
M_{x} \\
M_{y} \\
M_{x y}
\end{array}\right]=\left[\begin{array}{ccc}
D_{11} & D_{12} & 0 \\
D_{12} & D_{22} & 0 \\
0 & 0 & D_{66}
\end{array}\right]\left[\begin{array}{c}
\kappa_{x} \\
\kappa_{y} \\
\kappa_{x y}
\end{array}\right]=\boldsymbol{D} \boldsymbol{\kappa} .}
\end{aligned}
$$

The equivalent stiffness values of the honeycomb panel are calculated on the basis of the relationship between the equivalent stiffness and the strain energy of the unit cell using the RVE method and applying corresponding displacement boundary conditions [22]. The relationship between the equivalent stiffness matrix and the strain energy is expressed as follows:

$$
\frac{1}{2}\left(\left(\boldsymbol{\varepsilon}^{0}\right)^{\mathrm{T}}, \boldsymbol{\kappa}^{\mathrm{T}}\right)\left[\begin{array}{ll}
\boldsymbol{A} & \boldsymbol{B} \\
\boldsymbol{B}^{\mathrm{T}} & \boldsymbol{D}
\end{array}\right]\left(\begin{array}{c}
\boldsymbol{\varepsilon}^{0} \\
\boldsymbol{\kappa}
\end{array}\right)=\frac{U_{\text {cell }}}{V},
$$

where $V$ is the area of the unit cell, and $U_{\text {cell }}$ is the strain energy of the unit cell.

\subsection{Unit cell model and periodic boundary conditions}

The finite element method is used to solve the unit cell model as shown in Fig. 10. When building a finite element model, anisotropic and geometrically linear beam elements are used, e.g., the 3D three-node quadratic beam element, as shown in B32 in ABAQUS [24]. The beam section is

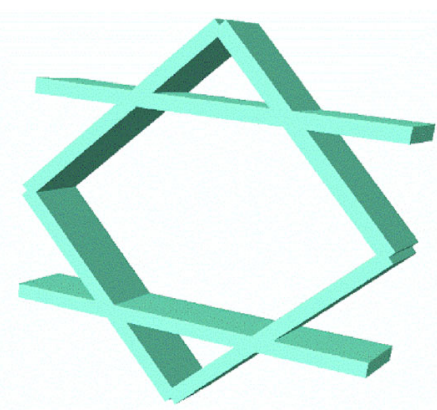

(b)

Fig. 10 Unit cell finite element model. (a) Unit cell model; (b) display of beam section. 
rectangular and the cross section size is $0.002 \mathrm{~m} \times 0.01 \mathrm{~m}$. Three local coordinate systems are established to define the material orientation given that the material is anisotropic. Material properties are shown in Table 1. The model includes 230 nodes, 116 beam elements, and eight boundary points.

Periodic boundary conditions are a standard tool in the computation of homogenized models for composites. The periodic boundary conditions in this study are in accordance with the work by Kueh and Pellegrino [25]. When a homogenized plate is subjected to uniform midplane strains $\varepsilon_{i j}^{0}$ and uniform mid-plane curvature $\kappa_{i j}$, the periodic boundary conditions for the changes in a displacement component $\Delta u_{i}$ and a rotation component $\Delta \theta_{i}$ of the corresponding nodes on boundaries can be defined as follows:

$$
\begin{aligned}
& \Delta u_{i}=\varepsilon_{i j}^{0} \times \Delta l_{j}, \\
& \Delta \theta_{i}=\kappa_{i j} \times \Delta l_{j},
\end{aligned}
$$

where $\Delta l_{j}$ is the distance between two corresponding boundary nodes. The corresponding displacement and rotation boundary conditions are summarized as follows:

$$
\begin{gathered}
u(a, y)-u(0, y)=\varepsilon_{x}^{0} \Delta l_{x}, \\
v(a, y)-v(0, y)=\gamma_{x y}^{0} \Delta l_{x} / 2, \\
w(a, y)-w(0, y)=-\kappa_{x y} y \Delta l_{x} / 2, \\
\theta_{x}(a, y)-\theta_{x}(0, y)=-\kappa_{x y} \Delta l_{x} / 2, \\
\theta_{y}(a, y)-\theta_{y}(0, y)=\kappa_{x} \Delta l_{x}, \\
\theta_{z}(a, y)-\theta_{z}(0, y)=0, \\
v(x, b)-v(x, 0)=\varepsilon_{y}^{0} \Delta l_{y},
\end{gathered}
$$

$$
\begin{gathered}
u(x, b)-u(x, 0)=\gamma_{x y}^{0} \Delta l_{y} / 2, \\
w(x, b)-w(x, 0)=-\kappa_{x y} x \Delta l_{y} / 2, \\
\theta_{y}(x, b)-\theta_{y}(x, 0)=\kappa_{x y} \Delta l_{y} / 2, \\
\theta_{x}(x, b)-\theta_{x}(x, 0)=-\kappa_{y} \Delta l_{y}, \\
\theta_{z}(x, b)-\theta_{z}(x, 0)=0 .
\end{gathered}
$$

According to Eqs. (1)-(3), the mid-plane strains and curvatures have six variables and the ABD matrix has eight unknowns. Therefore, eight periodic boundary conditions are imposed on the finite element model. In each case, one or two average strain/curvatures are set equal to one and all others equal to zero, and the corresponding strain energy $U_{\text {cell }}$ of the structure can be obtained. The corresponding unknowns are calculated by Eq. (4). For example, in the first analysis, $\varepsilon_{x}^{0}=1$ and $\varepsilon_{y}^{0}=\gamma_{x y}^{0}=\kappa_{x}=\kappa_{y}=\kappa_{x y}=0$ are set to calculate $A_{11}$, i.e., we can obtain $A_{11}=2 U_{\text {cell }} / V$. The periodic boundary conditions and corresponding equivalent stiffness calculation formulas are shown in Table 1.

The RVE analysis is completed to obtain the equivalent ABD matrices. For example, the process may be conducted in ABAQUS, where the strain energy of the structure can be accessed using the *ODB history output commands. Thus, the equivalent stiffness matrices for the fabricated composite Kagome panel are computed as follows:

$$
\begin{aligned}
& \boldsymbol{A}=\left[\begin{array}{ccc}
11.5 & 3 & 0 \\
3 & 3 & 0 \\
0 & 0 & 3
\end{array}\right] \times 10^{6} \mathrm{~N} / \mathrm{m}, \\
& \boldsymbol{D}=\left[\begin{array}{ccc}
95.9 & 24.7 & 0 \\
24.7 & 25.3 & 0 \\
0 & 0 & 25.2
\end{array}\right] \mathrm{N} \cdot \mathrm{m} .
\end{aligned}
$$

Table 1 Periodic boundary conditions and corresponding equivalent stiffness of Kagome honeycomb

\begin{tabular}{llcc}
\hline Stiffness & \multicolumn{1}{c}{ Initial strain } & Boundary displacement and rotation & Stiffness formula \\
\hline$A_{11}$ & $\boldsymbol{\varepsilon}^{0}=[1,0,0]^{\mathrm{T}}, \boldsymbol{\kappa}=[0,0,0]^{\mathrm{T}}$ & $u(a, y)-u(0, y)=a, v(x, b)-v(x, 0)=0$ & $A_{11}=2 U_{\text {cell }} / V$ \\
$A_{22}$ & $\boldsymbol{\varepsilon}^{0}=[0,1,0]^{\mathrm{T}}, \boldsymbol{\kappa}=[0,0,0]^{\mathrm{T}}$ & $u(a, y)-u(0, y)=0, v(x, b)-v(x, 0)=b$ & $A_{22}=2 U_{\text {cell }} / V$ \\
$A_{66}$ & $\boldsymbol{\varepsilon}^{0}=[0,0,1]^{\mathrm{T}}, \boldsymbol{\kappa}=[0,0,0]^{\mathrm{T}}$ & $v(a, y)-v(0, y)=a / 2, u(x, b)-u(x, 0)=b / 2$ & $A_{66}=2 U_{\text {cell }} / V$ \\
$A_{12}$ & $\boldsymbol{\varepsilon}^{0}=[1,1,0]^{\mathrm{T}}, \boldsymbol{\kappa}=[0,0,0]^{\mathrm{T}}$ & $u(a, y)-u(0, y)=a, v(x, b)-v(x, 0)=b$ & $A_{12}=\left(2 U_{\text {cell }} / V-A_{11}-A_{22}\right) / 2$ \\
$D_{11}$ & $\boldsymbol{\varepsilon}^{0}=[0,0,0]^{\mathrm{T}}, \boldsymbol{\kappa}=[1,0,0]^{\mathrm{T}}$ & $\theta_{y}(a, y)-\theta_{y}(0, y)=a, \theta_{x}(x, b)-\theta_{x}(x, 0)=0$ & $D_{11}=2 U_{\text {cell }} / V$ \\
$D_{22}$ & $\boldsymbol{\varepsilon}^{0}=[0,0,0]^{\mathrm{T}}, \boldsymbol{\kappa}=[0,1,0]^{\mathrm{T}}$ & $\theta_{y}(a, y)-\theta_{y}(0, y)=0, \theta_{x}(x, b)-\theta_{x}(x, 0)=-b$ & $D_{22}=2 U_{\text {cell }} / V$ \\
$D_{66}$ & $\boldsymbol{\varepsilon}^{0}=[0,0,0]^{\mathrm{T}}, \boldsymbol{\kappa}=[0,0,1]^{\mathrm{T}}$ & $w(a, y)-w(0, y)=-a y / 2, w(x, b)-w(x, 0)=-b x / 2$, & $D_{66}=2 U_{\text {cell }} / V$ \\
$D_{12}$ & $\theta_{x}(a, y)-\theta_{x}(0, y)=-a / 2, \theta_{y}(x, b)-\theta_{y}(x, 0)=b / 2$, & $D_{12}=\left(2 U_{\text {cell }} / V-D_{11}-D_{22}\right) / 2$ \\
\hline
\end{tabular}




\subsection{Finite element simulation of the equivalent plate}

To verify the equivalent stiffness of the honeycomb panel, the Kagome honeycombs with different combinations are analyzed in accordance with two models, namely, the discrete structure and the equivalent plate under different boundary conditions and different loads. We perform inplane stiffness verification. The material parameters used in the discrete model are shown in Section 2.3. Specific conditions are summarized as follows: The bottom side of the model is fixed by a multipoint constraint in ABAQUS, and the upper side is applied with a concentrated load by multipoint constraint. The axial displacements of the two models under this loading condition are shown in Table 2. The comparative results show that the equivalent model has high accuracy in terms of in-plane stiffness, indicating that the RVE method can effectively homogenize the Kagome honeycomb panel as a continuum plate.

The specific conditions in the verification of out-ofplane stiffness are as follows: The four sides of the model are fixed and a concentrated load is applied at the center point of the model. The center point displacement of the two models under this condition is shown in Table 3 . The comparative results show a certain error between the discrete solution and the equivalent solution when the number of the unit cells is relatively small, and the error decreases with the increase in unit cell combinations. When the structure has more RVEs, its boundary effect is weakened. Thus, the predicted value is relatively close to the value of the discrete analysis. The comparison of the inplane and out-of-plane analysis illustrates the accuracy of the equivalent stiffness.

\section{Experiment}

\subsection{Compressive response of Kagome honeycombs}

Compression tests are performed on a testing machine to explore the in-plane stiffness of Kagome honeycomb panels with the displacement rate of $0.50 \mathrm{~mm} / \mathrm{min}$. The experimental samples include $2 \times 1$ and $3 \times 2$ Kagome honeycombs fabricated using the present method in Section 2 . The $2 \times 1$ Kagome honeycomb sample has a length of $93.8 \mathrm{~mm}$ and a width of $48.6 \mathrm{~mm}$. The $3 \times 2$ Kagome honeycomb sample has a length of $139 \mathrm{~mm}$ and a width of $93.8 \mathrm{~mm}$. The height of all the samples is $10 \mathrm{~mm}$. At least three test samples are used for each configuration. The typical load-displacement curve of $2 \times 1$ Kagome honeycomb is plotted in Fig. 11. In the $O-A$ segment in Fig. 11, the force-displacement curve is linear, and a certain error is found between the results of the finite element equivalent model and the experiment, but the basic trend remains the same. The stiffness of the honeycomb begins to decline after reaching Point $A$ in the experiment. In addition, the load-carrying capacity of the honeycomb begins to decline sharply at Point $C$ when the cell wall is completely broken.

The load-displacement curve of $3 \times 2$ Kagome honeycomb is plotted in Fig. 12. The linear-elastic deformation is completed when initial damage occurs. The experimental results are in good agreement with the results of the finite element equivalent model. Then, some fluctuations are found in the load-displacement curve subsequent to the peak load. This finding implies that the Kagome honeycomb has gradually failed during the

Table 2 Axial displacement of Kagome honeycombs under in-plane loading

\begin{tabular}{|c|c|c|c|}
\hline \multirow{2}{*}{ Unit cell combination } & \multicolumn{2}{|c|}{ Axial displacement $/ \mathrm{m}$} & \multirow{2}{*}{ Error $/ \%$} \\
\hline & Discrete honeycomb & Equivalent plate & \\
\hline$\overline{2 \times 1}$ & $-3.47 \times 10^{-4}$ & $-3.40 \times 10^{-4}$ & 1.8 \\
\hline $3 \times 2$ & $-2.57 \times 10^{-4}$ & $-2.52 \times 10^{-4}$ & 1.9 \\
\hline $5 \times 3$ & $-2.83 \times 10^{-4}$ & $-2.78 \times 10^{-4}$ & 1.8 \\
\hline $11 \times 10$ & $-1.76 \times 10^{-4}$ & $-1.73 \times 10^{-4}$ & 1.7 \\
\hline
\end{tabular}

Table 3 Center point displacement of Kagome honeycombs under bending

\begin{tabular}{|c|c|c|c|}
\hline \multirow{2}{*}{ Unit cell combination } & \multicolumn{2}{|c|}{ Max displacement/m } & \multirow{2}{*}{ Error/\% } \\
\hline & Discrete honeycomb & Equivalent plate & \\
\hline $3 \times 2$ & $-1.09 \times 10^{-3}$ & $-1.21 \times 10^{-3}$ & 9.9 \\
\hline $2 \times 4$ & $-0.81 \times 10^{-3}$ & $-0.85 \times 10^{-3}$ & 4.9 \\
\hline $5 \times 3$ & $-0.42 \times 10^{-3}$ & $-0.43 \times 10^{-3}$ & 2.4 \\
\hline $11 \times 10$ & $-1.30 \times 10^{-2}$ & $-1.32 \times 10^{-2}$ & 1.5 \\
\hline
\end{tabular}



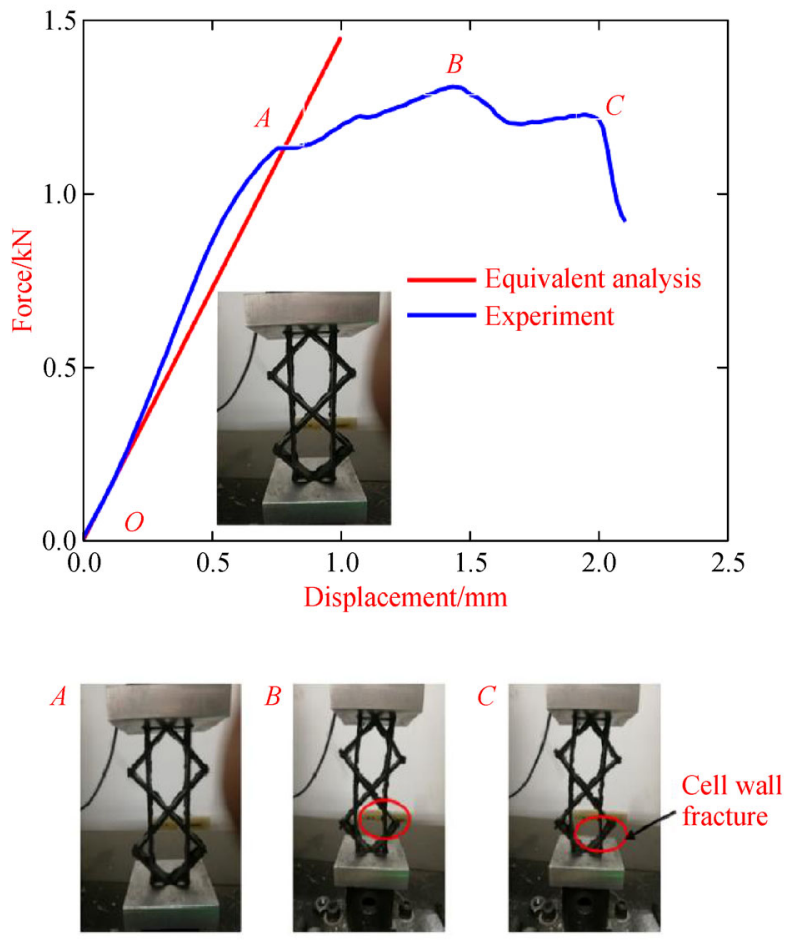

Fig. 11 Compression response of $2 \times 1$ Kagome honeycomb: Linear elastic deformation (Point $A$ ), local damage (Point $B$ ), and cell wall fracture (Point $C$ ).

testing. The load-carrying capacity of the honeycomb has begun to decline sharply at Point $E$ when the cell wall is completely broken.

The honeycomb compression responses with $2 \times 1$ and $3 \times 2$ combinations are observed, and the test results verify the in-plane stiffness obtained by RVE. Moreover, the theoretical and experimental results agree well as the number of unit cells increases.

\subsection{Bending response of Kagome honeycombs}

Three-point bending tests are performed on a testing machine to explore the out-of-plane stiffness of Kagome honeycombs with a displacement rate of $1 \mathrm{~mm} / \mathrm{min}$. The experimental samples include $3 \times 2$ and $4 \times 2$ Kagome honeycombs. The length, width, and height of the $3 \times 2$ honeycomb are the same with the sample described in the previous section. The length of $4 \times 2$ Kagome honeycomb samples is $184.2 \mathrm{~mm}$ and the width is $93.8 \mathrm{~mm}$. The height of all the samples is $10 \mathrm{~mm}$. At least three samples are tested for each combination. The load-displacement curves are plotted in Figs. 13(a) and 13(b) for $4 \times 2$ and $3 \times 2$ Kagome honeycombs, respectively. From Point $O$ to Point $F$ in Fig. 13(a) and Point $O$ to Point $G$ in Fig. 13(b), the honeycomb behaves approximately linear-elastic.
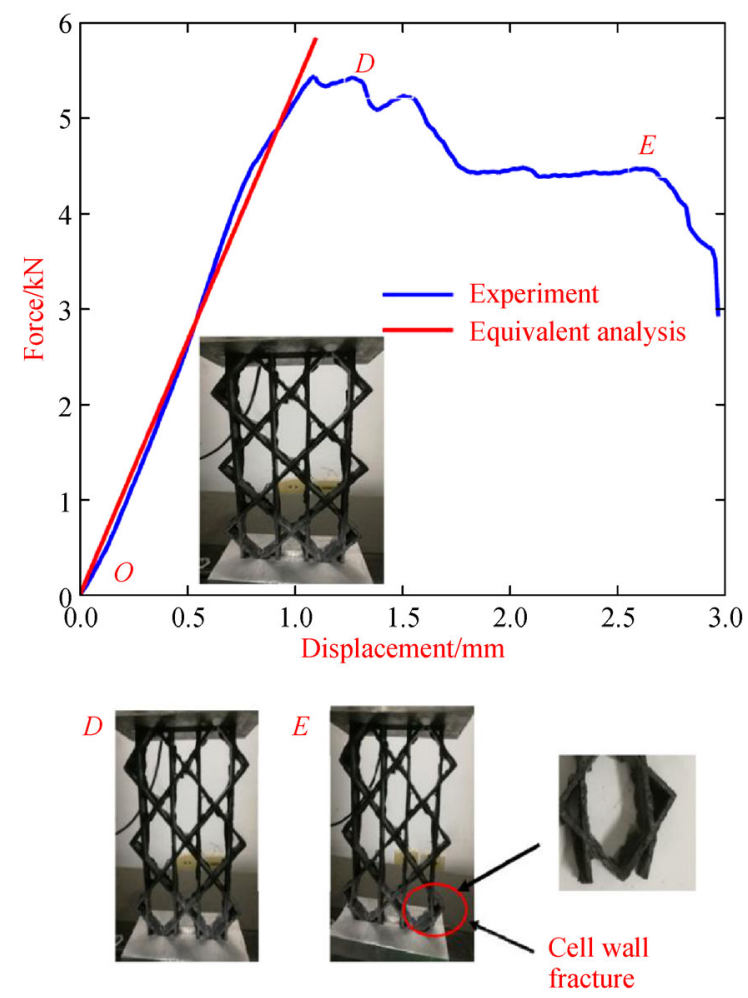

Fig. 12 Compression response of $3 \times 2$ Kagome honeycombs: Local damage (Point $D$ ) and cell wall fracture (Point $E$ ).

Subsequently, the stiffness of the honeycomb begins to drop. The results of the finite element equivalent model have some errors with the experimental results, but the overall trend is consistent. The reason for this phenomenon may be the insufficiently large number of unit cells in the experiments, resulting in a boundary effect and affecting the accuracy of the equivalent analysis. Installation error and manufacturing imperfection may also contribute to the error.

\section{Conclusions}

A novel modular mold is designed to manufacture CFRP Kagome honeycomb panel. The designed mold can be assembled by the boundary blocks and the internal blocks to form a fiber placement gap, which is beneficial to the placement of the fiber bundle. This mold can be assembled freely to manufacture Kagome honeycombs with different unit cell combinations and can achieve integral forming of the Kagome honeycombs.

Moreover, the effects of fiber placement methods on structural mechanical properties have been considered in this study. The Kagome honeycombs manufactured through two different fiber placement methods are 


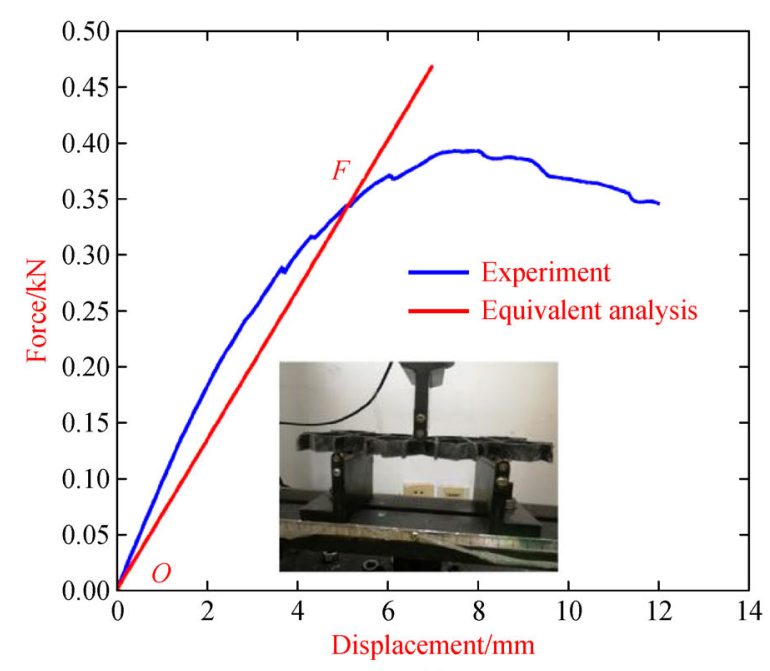

(a)

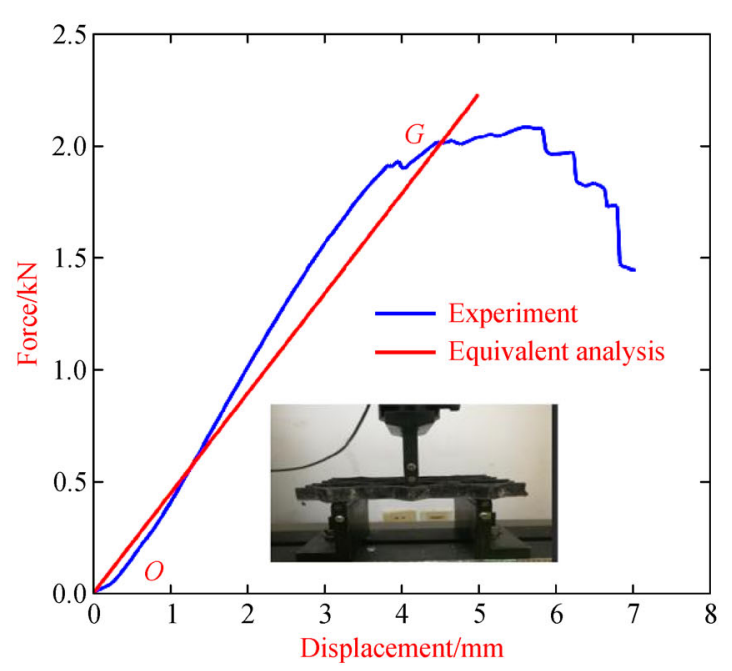

(b)

Fig. 13 Bending response of Kagome honeycombs. (a) $4 \times 2$ Kagome honeycombs; (b) $3 \times 2$ Kagome honeycombs.

compared by compression and bending testing. The test results show that the fiber placement method ensuring continuous fiber connection at the intersection can fully use the mechanical properties of fibers. Therefore, the resulting honeycomb has better mechanical properties. In addition, to prevent reduction in the quality of the Kagome honeycombs due to the excessive resin content in the fiber placement gap of the mold and the insufficient fiber content, the vacuum bag assisted curing process is conducted to carry out the compaction operation after the final placement is completed.

A homogenization analysis model is developed using RVE to calculate the equivalent in-plane and bending stiffness of orthotropic composite Kagome honeycomb panel. The expressions of eight stiffness coefficients and the periodic boundary conditions in the homogenization model using RVE are presented. The developed model is shown to be reliable by comparison with the discrete finite element simulation of the composite Kagome honeycomb panels under different load conditions, including in-plane and out-of-plane analyses. In addition, the Kagome honeycombs with different combinations are tested experimentally, and the results of the finite element equivalent model are in good agreement with the experimental results. Moreover, as the number of unit cells increases, the accuracy of the equivalent stiffness of the honeycomb calculated by RVE increases. The comparison also indicates that the Kagome honeycombs made by the designed mold have high quality.

Acknowledgements This work was partially supported by the National Natural Science Foundation of China (Grant Nos. 51975087, 51790172, 51675082, and 51505064) and the Fundamental Research Funds for the Central Universities (Grant No. DUT17ZD207). These supports are gratefully appreciated.

Open Access This article is licensed under a Creative Commons
Attribution 4.0 International License, which permits use, sharing, adaptation, distribution and reproduction in any medium or format, as long as you give appropriate credit to the original author(s) and the source, provide a link to the Creative Commons licence, and indicate if changes were made.

The images or other third party material in this article are included in the article's Creative Commons licence, unless indicated otherwise in a credit line to the material. If material is not included in the article's Creative Commons licence and your intended use is not permitted by statutory regulation or exceeds the permitted use, you will need to obtain permission directly from the copyright holder.

To view a copy of this licence, visit http://creativecommons.org/licenses/ by $/ 4.0 \%$.

\section{References}

1. Ashby M F. Drivers for material development in the 21 st century. Progress in Materials Science, 2001, 46(3-4): 191-199

2. Evans A G. Lightweight materials and structures. MRS Bulletin, 2001, 26(10): 790-797

3. Fan H, Zeng T, Fang D N, et al. Mechanics of advanced fiber reinforced lattice composites. Acta Mechanica Sinica, 2010, 26(6): 825-835

4. Restrepo D, Mankame N D, Zavattieri P D. Programmable materials based on periodic cellular solids. Part I: Experiments. International Journal of Solids and Structures, 2016, 100-101: 485-504

5. Fan H, Yang L, Sun F, et al. Compression and bending performances of carbon fiber reinforced lattice-core sandwich composites. Composites. Part A, Applied Science and Manufacturing, 2013, 52: $118-125$

6. Galletti G G, Vinquist C, Es-Said O S. Theoretical design and analysis of a honeycomb panel sandwich structure loaded in pure bending. Engineering Failure Analysis, 2008, 15(5): 555-562

7. Zhang G, Ma L, Wang B, et al. Mechanical behaviour of CFRP sandwich structures with tetrahedral lattice truss cores. Composites. Part B, Engineering, 2012, 43(2): 471-476

8. Deshpande V S, Ashby M F, Fleck N A. Foam topology: Bending 
versus stretching dominated architectures. Acta Materialia, 2001, 49(6): 1035-1040

9. Totaro G, Gürdal Z. Optimal design of composite lattice shell structures for aerospace applications. Aerospace Science and Technology, 2009, 13(4-5): 157-164

10. Niu B, Yan J, Cheng G. Optimum structure with homogeneous optimum cellular material for maximum fundamental frequency. Structural and Multidisciplinary Optimization, 2009, 39(2): 115132

11. Deshpande V S, Fleck N A. Collapse of truss core sandwich beams in 3-point bending. International Journal of Solids and Structures, 2001, 38(36-37): 6275-6305

12. Zuo X Q, Zhou Y, Mei J. Structure and compressive behavior of Fe25Cr5Al metallic honeycomb fabricated by powder extrusion molding and sintering. Materials for Mechanical Engineering, 2006, 30(10): 34-35 (in Chinese)

13. Vasiliev V V, Barynin V A, Rasin A F. Anisogrid lattice structures - Survey of development and application. Composite Structures, 2001, 54(2-3): 361-370

14. Sorrentino L, Marchetti M, Bellini C, et al. Design and manufacturing of an isogrid structure in composite material: Numerical and experimental results. Composite Structures, 2016, 143: 189-201

15. Han D, Tsai S W. Interlocked composite grids design and manufacturing. Journal of Composite Materials, 2003, 37(4): $287-$ 316

16. Fan H, Meng F, Yang W. Sandwich panels with Kagome lattice cores reinforced by carbon fibers. Steel Construction, 2008, 81(4):
533-539

17. Wang A J, McDowell D L. Yield surfaces of various periodic metal honeycombs at intermediate relative density. International Journal of Plasticity, 2005, 21(2): 285-320

18. Niu B, Wang B. Directional mechanical properties and wave propagation directionality of Kagome honeycomb structures. European Journal of Mechanics. A, Solids, 2016, 57: 45-58

19. Cheng G, Cai Y, Xu L. Novel implementation of homogenization method to predict effective properties of periodic materials. Acta Mechanica Sinica, 2013, 29(4): 550-556

20. Cai Y, Xu L, Cheng G. Novel numerical implementation of asymptotic homogenization method for periodic plate structures. International Journal of Solids and Structures, 2014, 51(1): 284292

21. Peng B, Yu W. A micromechanics theory for homogenization and dehomogenization of aperiodic heterogeneous materials. Composite Structures, 2018, 199: 53-62

22. Liu S, Su W. Effective couple-stress continuum model of cellular solids and size effects analysis. International Journal of Solids and Structures, 2009, 46(14-15): 2787-2799

23. Whitney J M. Structural Analysis of Laminated Anisotropic Plates. New York: CRC Press, 1987, 23-26

24. Dassault Systemes Simulia, Inc. Abaqus Analysis User's Manual. Version 6.11. 2011

25. Kueh A, Pellegrino S. ABD matrix of single-ply triaxial weave fabric composites. In: Proceedings of the 48th AIAA/ASME/ASCE/ AHS/ASC Structural Dynamics and Materials Conference. Honolulu: AIAA, 2007, 5498-5514 